\title{
Sensorless Manipulation Using Transverse Vibrations of a Plate
}

\author{
Karl-Friedrich Böhringer
Robotics \& Vision Laboratory
Dept. of Computer Science
Cornell University \\ Ithaca, NY 14853-7501
}

\author{
Vivek Bhatt
}

Kenneth Y. Goldberg

Dept. of Computer Science

University of

Southern California

Los Angeles, CA 90089-0273

http://www.cs.cornell.edu/Info/People/karl/VibratoryAlign/home.html

\begin{abstract}
The existing industrial parts feeders move the parts through a sequence of mechanical filters that reject parts in unwanted orientations. In this paper we develop a new setup that uses a different vibratory mechanism to systematically manipulate parts, by actively orienting and localizing them. The idea is to generate and change dynamic modes for a plate by varying the applied frequency of oscillation. Depending on the node shapes of the plate for these frequencies, the position and orientation of the parts can be controlled. We develop an analysis of the underlying dynamics, and show that it can be used to predict the behavior of objects placed on the vibrating plate. Using this analysis, we propose that the applied frequencies can be automatically sequenced to obtain a "sensorless" strategy for manipulating a given object.
\end{abstract}

\section{Introduction}

Since it is extremely costly to maintain part order throughout the manufacture cycle, for example by keeping parts in pallets, parts are often delivered in bags or boxes, from where they must be picked out and sorted. A parts feeder is a machine that orients such parts before they are fed to an assembly station. Currently, the design of parts feeders is a black art that is responsible for up to $30 \%$ of the cost and $50 \%$ of workcell failures $[15,3,8,21,22]$. "The real problem is not part transfer but part orientation.", Frank Riley, Bodine Corporation [19, p.316, his italics]. Thus although part feeding accounts for a large portion of assembly cost, there is not much scientific basis for automating the process.

The most common type of feeder is the vibratory bowl feeder, where parts in a bowl are vibrated with a rotary motion so that they climb a helical track. As they climb, a sequence of baffles and cutouts in the track create a mechanical "filter" that causes parts in all but one orientation to fall back into the bowl for another attempt at running the gauntlet $[3,19,20]$. To improve feedrate, it is sometimes possible to design the track so as to mechanically rotate parts into a desired orientation (this is called conversion). Related methods use centrifugal forces [8], reciprocating forks, or belts to move parts through the filter [18].

Sony's APOS parts feeder [13] uses an array of nests (silhouette traps) cut into a vibrating plate. The nests and the vibratory motion are designed so that the part will remain in the nest only in a particular orientation. By tilting the plate and letting parts flow across it, the nests eventually fill up with parts in the desired orientation. Although the vibratory motion is under software control, specialized mechanical nests must be designed for each part [14].

Singer and Seering [22] proposed several designs for parts feeders where programmed vibration was used to drive parts into a stable configuration. These methods can be useful for bringing parts into one of several poses where its center of mass is as low as possible.

Despite their popularity, these vibratory feeders have some disadvantages:

1. Parts may get wedged or entangled in filters.

2. Parts may get damaged when dropping back into the bowl, or worn by repeated rejections.

3. Each filter reduces the feeding rate.

4. The filters have to be redesigned for each new part geometry, a task that usually requires skillful work by human experts. ${ }^{1}$

In the early 1980's, several researchers used sensors to determine the pose of parts delivered by a vibratory

\footnotetext{
${ }^{1}$ Caine [4] presented an experimental CAD system that assists the construction of track filters for vibratory bowl feeders.
} 
track [18]. Sensors such as tactile probes [10, 25], photocells [11], fiberoptic sensors [16], and machine vision systems [12, 23] were employed. Once part pose was determined, air-jets and trapdoors were used to group parts in similar poses.

In this paper, we explore how controlled vibration can be used for a new setup to systematically feed planar parts. The idea is to generate and change dynamic modes in a plate by varying applied frequencies. Depending on the frequency of vibration and the boundary conditions, nodes of different shapes are formed (this was experimentally studied by Chladni [6]). If planar parts are put on this vibrating plate, they move to the node, and end up in a stable orientation. We develop an analysis whereby given the shape of the node, and the part geometry, the final orientation can be predicted. For our device, we further propose a "sensorless" strategy for part manipulation [7], using the theory originally developed for feeding parts using parallel-jaw grippers [9], which was recently extended to arrays of microactuators (Böhringer et al. [2]). Working independently, Will and Liu are also exploring the idea of using a dense array of microactuators to create programmable 'force fields' that can be used to position and orient small parts. Their simulator and initial designs for such fields are reported in [26].

The main characteristics of our device are:

- simple design, with no mechanical filters (addressing disadvantages $1-3$ )

- programmability (addressing problem 4).

Section 2 describes the design of our devices, and the performed experiments. In Section 3 we investigate the dynamics of small particles on the plate to deduce the approximate nature of the effective force field on the plate. The behavior of objects in a planar force field is discussed in Section 4. In Section 5, this model is used to predict the stable rest configurations for parts on the vibrating plate, and the predictions are compared with experimental results. Section 6 demonstrates how our device can be programmed for sensorless manipulation.

\section{Experimental Observations}

\subsection{Setup and Calibration}

Figures 1 and 2 are schematic of the experimental setup, which consists of an aluminum plate forced to oscillate in two different configurations. The shaker is a commercially available ${ }^{2}$ electrodynamic vibration

\footnotetext{
${ }^{2}$ Model VT-100G, Vibration Test Systems, Akron, OH, USA.
}

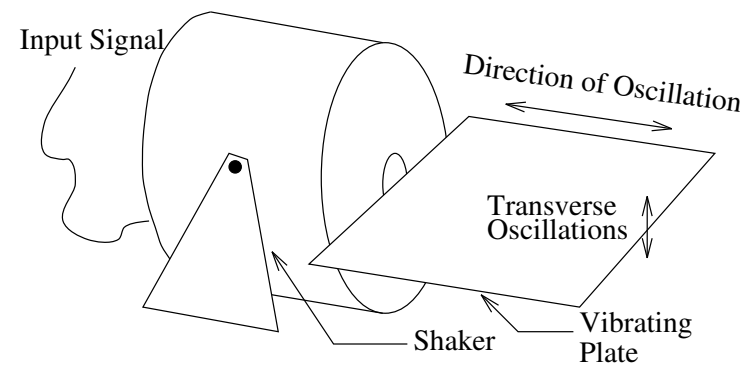

Figure 1: Schematic of experimental setup 1.

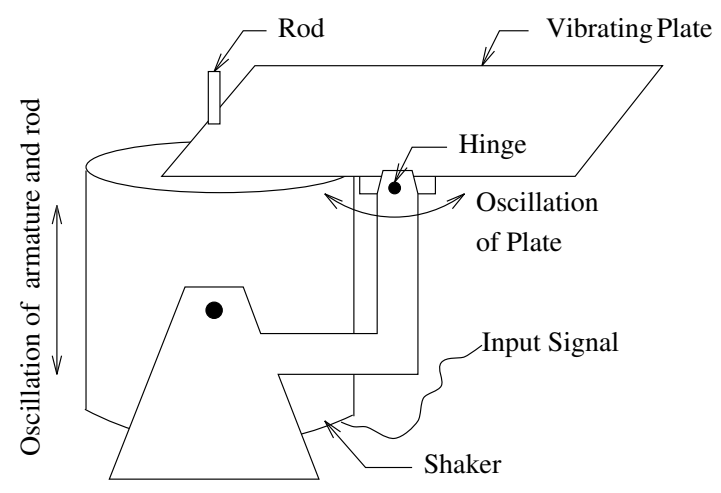

Figure 2: Schematic of experimental setup 2.

generator, with a linear travel of $0.02 \mathrm{~m}$, and capable of producing a force of up to $500 \mathrm{~N}$. The input signal, specifying the waveform corresponding to the desired oscillations, is fed to a single coil armature, which moves in a constant field produced by a ceramic permanent magnet in a center gap configuration.

In the first configuration (Figure 1), the plate is attached to the shaker armature such that it is forced to vibrate in the longitudinal direction. For low amplitudes and frequencies, the plate moves longitudinally with no perceptible transverse vibrations. However, as the frequency of oscillations is increased, transverse vibrations of the plate become more pronounced. The resulting motion is similar to the forced transverse vibration of a rectangular plate, clamped on one edge and free along the other three sides.

The nodes for these transverse oscillations can either be obtained theoretically (Rayleigh [17], Timoshenko [24]), or experimentally using the technique originally pioneered by Chladni [6]. By sprinkling small sized particles (Chladni used sand, we use Urad lentils to get a better contrast on video) on a vibrating surface, the nodes can be experimentally identified as the regions where the particles tend to collect. The dynamics of "collecting" at the nodes, is important in determining the effective force field that leads to the orienting and localization effect of our device, and is discussed in detail in Section 3. 

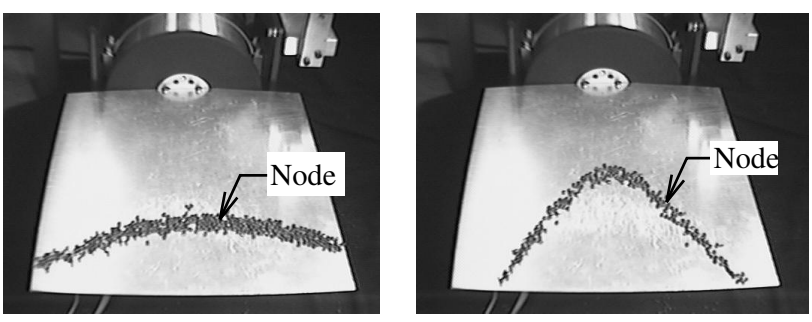

Figure 3: Experimentally determined nodes, at $60 \mathrm{~Hz}$ (left) and $100 \mathrm{~Hz}$ (right), for the setup in Figure 1.
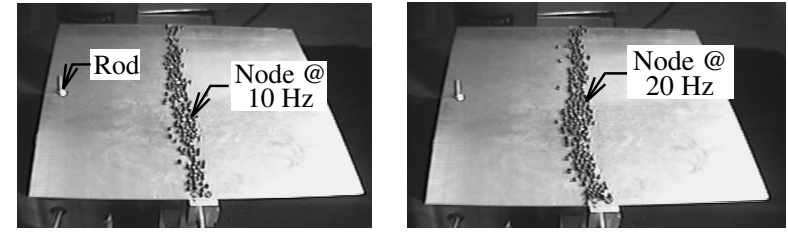

Figure 4: Experimentally determined nodes at $10 \mathrm{~Hz}$ (left) and $20 \mathrm{~Hz}$ (right) for the setup in Figure 2.

For the configuration in Figure 1, the location and shape of the node depends on the frequency of vibration. Figure 3 shows the experimental determination of the nodes for frequencies of $60 \mathrm{~Hz}$ and $100 \mathrm{~Hz}$.

The second configuration for the plate (Figure 2), forces the plate to undergo transverse vibrations such that the resulting shape of the node, and its location, are independent of the forcing frequency. The plate is hinged about an axis situated midway between, and parallel to, two of its sides. A rod connected to the armature of the shaker forces the plate to an oscillatory motion about the hinged axis. As expected, experimental determination shows that except for a slight distortion due to the effect of clamping at the rod, the node lines up with the hinge axis (Figure 4).

The second setup is run at lower frequencies, to ensure that only the mode where the plate oscillates about the hinge axis is excited. If we increase the operating frequency, modes corresponding to transverse vibration of a plate, clamped at the point of attachment to the rod and the hinged ends, become dominant, and the node shape gets complicated. This effect can be seen at $20 \mathrm{~Hz}$ (Figure 4), where the node shows a tendency to get "pulled" towards the point where the plate is clamped to the rod.

\subsection{Behavior of Planar Parts}

If we put planar shapes on the vibrating surface, there is a marked tendency for them to move towards the node and end up in one of a finite number of stable orientations. We observe the following features over a wide range of frequencies in both the experimental setups:

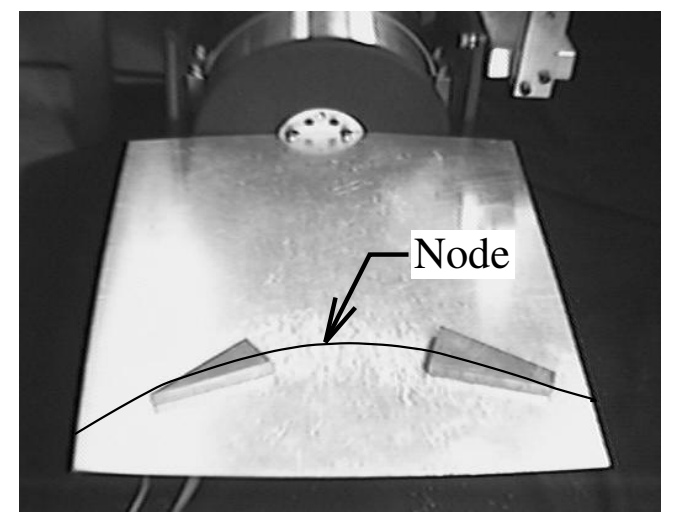

Figure 5: Stable position of planar parts, at a frequency of $60 \mathrm{~Hz}$. The node is marked according to Figure 3.

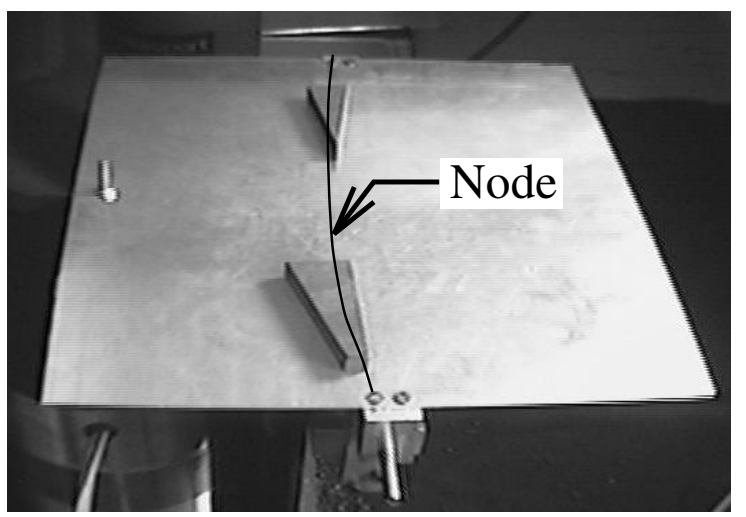

Figure 6: Stable position of planar parts, at a frequency of $20 \mathrm{~Hz}$. The node is marked according to Figure 4.

- From all initial positions on the plate, the objects move towards the node. They end up in a stable position around some point on the node, which depends on the initial position of the object.

- As the object approaches the node (as we show later, after some portion of it crosses the node), there is a tendency for it to rotate until it reaches one of a finite number of stable orientations.

Figure 5 shows two planar shapes, a triangle and a trapezoid, after they have reached their stable position and orientation for the setup in Figure 1. To better illustrate the orienting effect, the curve showing the node has been drawn by hand. Figure 6 , similarly shows the stable position of the planar parts for the second setup (Figure 6).

Over the large number of experimental runs performed, there are a couple of qualitative observations describing the ease and speed with which the parts get into a stable configuration:

- At higher frequencies of oscillation, both the velocity of the part towards the node, and the rate of orientation, are relatively faster. 
- Objects with a higher degree of rotational asymmetry get into a stable orientation more easily.

Although the location of the node is better identified in the second setup, the lower operating frequencies make the localization of the part at the node, and the corresponding orienting behavior, much slower. These trade-offs are design considerations that need to be investigated separately.

\section{$3 \quad$ Identifying the Force Field}

The underlying dynamics that causes the objects placed in a vibrating surface to move towards the node give rise to an effective force field. In order to develop a theory for using our device as a viable method for sensorless manipulation, it is important to determine the genesis and variation of this force field over the vibrating plate.

When particles are spread on a vibrating surface, they collect at the nodes, resulting in patterns known as Chladni figures (after Chladni [6]). Rayleigh [17] describes the motion of the particles towards the nodes in the following words - "the movement to the nodes is irregular in its character. If a grain be situated elsewhere than at a node, it is made to jump by a sufficiently vigorous transverse vibration. The result may be a movement either towards or from a node; but after a succession of such jumps the grain ultimately finds its way to a node".

The forces that cause the particles to move to the node act on any object placed on the vibrating surface, generating an effective force field. The underlying dynamics of this phenomenon is still poorly understood. In the Appendix we give an approach towards an analytical model for the more tractable case of the planar motion of a particle bouncing on a string in transverse vibration.

Results of our experiments indicate that the forces at each location of the plate can be thought of as proportional to the amplitude of vibration, and perpendicular to the sinusoidal "envelope" surface of the oscillating plate. They are also proportional to the oscillation frequency, and the coefficient of restitution. The first order approximation near the nodes can be used to show that the force field is perpendicular to and points towards the node, and varies linearly with the distance from the node. This information is enough to predict the final stable orientation for the parts. For simulating the field far from the node, we approximate its magnitude by a sine variation normal to the node curve.

\section{Motion of Parts on the Plate}

The case of general objects on the plate is more complicated than individual particles, because the determination of the point on the object that undergoes impact, and the resulting impulses, are both difficult problems to solve. For our analysis, we ignore effects such as rolling and tilting of the parts and assume that the contact geometry remains constant over the impacts.

Instead of dealing with general parts, for which a more complicated formulation would be required, we look at planar parts for which deriving the stability properties is relatively straightforward.

We can consider the planar parts as a conglomeration of "particles", each of which interacts with the plate and experiences the effective force field discussed in Section 3. The forces have to be averaged out over the area of contact, giving a specific force (per unit area), $f$, that acts on all points of the planar object.

Let $\mathcal{P}$ be the planar part in contact with the vibrating plate $\mathcal{V}$, and $c$ denote the center of mass of $\mathcal{P}$, with $\mathcal{S}$ its surface in contact with $\mathcal{V}$; the total force $F$ and the moment $M$ around $c$ can be obtained by integrating the force field $f$ over the contact surface $\mathcal{S}$,

$$
\begin{aligned}
F & =\int_{\mathcal{S}} f d A \\
M & =\int_{\mathcal{S}}(r-c) \times f d A
\end{aligned}
$$

We have made a series of assumptions to suggest that a force field exists for parts on a planar plate. Our experimental results indicate that they are good engineering assumptions when we observe the system over time, due to an averaging effect caused by the vibration of the plate. An "exact" modeling of the impact dynamics between part and plate is intractable as well as unnecessary for our purposes.

\section{Prediction of Rest Configurations}

Consider a part $\mathcal{P}$ on the vibrating plate $\mathcal{V}$. A rest configuration is a placement of $\mathcal{P}$ on $\mathcal{V}$ such that $\mathcal{P}$ remains stationary, and stable with respect to small disturbances. In a rest configuration, the force and moment acting on $\mathcal{P}$ are in equilibrium. This equilibrium condition is met when the force $F$ and the moment $M$ (Eqs. $(1,2)$, Section 4) are zero:

$$
\begin{aligned}
F & =\int_{\mathcal{S}} f d A=0 \\
M & =\int_{\mathcal{S}}(r-c) \times f d A=0
\end{aligned}
$$




\section{$5.1 \quad$ Example}

Consider a case similar to Figure 6 where the vibrating plate $\mathcal{V}$ lies in the $x$-y-plane, and its node is a straight line coinciding with the $y$-axis. Further assume that the force field generated by $\mathcal{V}$ can be described as follows:

$$
f(x, y)= \begin{cases}(1,0) & \text { if } x<0 \\ (0,0) & \text { if } x=0 \\ (-1,0) & \text { if } x>0\end{cases}
$$

Under these assumptions the equilibrium condition (Eqs. 3, 4) simplifies to:

$$
\begin{array}{rll}
\mathcal{S}_{l} & =\mathcal{S}_{r} \\
c_{l}-c_{r} & \perp & y \text {-axis }
\end{array}
$$

$\mathcal{S}_{l}$ and $\mathcal{S}_{r}$ are the part of $\mathcal{S}$ on the left and right of the $y$-axis, respectively, and $c_{l}$ and $c_{r}$ are their respective centers of mass. This means that a part is in equilibrium if the areas of contact left and right of the $y$-axis are equal, and if the line connecting the left and right center of area is perpendicular to the $y$-axis (see Figure 7).

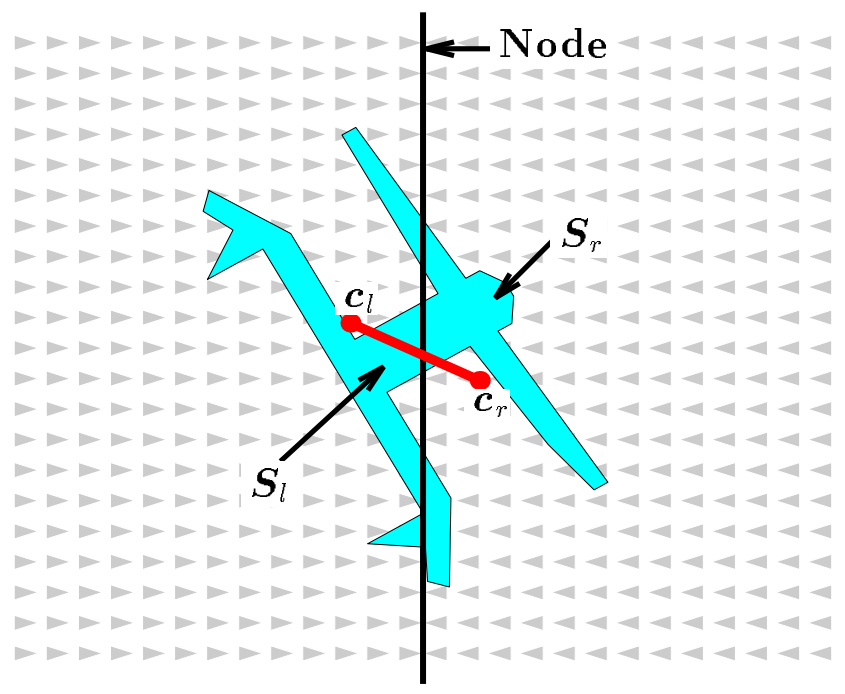

Figure 7: Equilibrium Condition

Force and moment equilibrium is not sufficient in practice for a part to come to rest. An equilibrium may be metastable, i.e. small disturbances of the part position or orientation may cause the part to move away from equilibrium. To understand this effect better consider a part $\mathcal{P}$ in orientation $\theta$ in force equilibrium $(F=0)$. For each orientation $\theta$ we can determine the resulting moment $M$ on $\mathcal{P}$. Note that for given $\theta, M$ is determined uniquely. Thus we obtain a map from orientation $\theta$ to moment $M$. We call this map the turn function $t_{\mathcal{P}}: \mathbb{S}^{1} \rightarrow \mathbb{R}$ of part $\mathcal{P}$, because it describes the relationship between part orientation and its instantaneous rotation. If $t_{\mathcal{P}}(\theta)>0$ there is a moment acting on $\mathcal{P}$ that will cause a counterclockwise instantaneous rotation. $t_{\mathcal{P}}(\theta)<0$ corresponds to a clockwise instantaneous rotation. Roots of the turn function correspond to equilibrium configurations. It can be shown (see Böhringer et al. [1]) that an equilibrium is stable if $t_{\mathcal{P}}$ changes from positive to negative at $\theta$, otherwise it is metastable. Figures $8 \mathrm{a}$ and $\mathrm{b}$ show the part and its turn function for a force field $f$ as defined above.

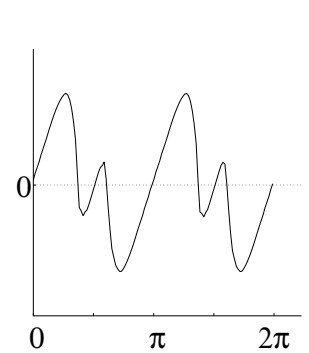

(a) Turn Function $t_{\mathcal{P}}$

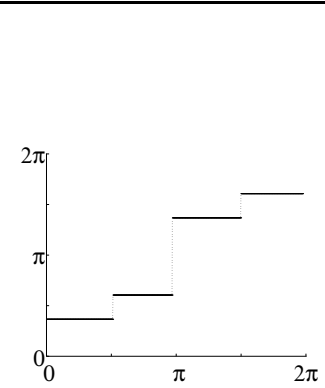

(c) Alignment Fct. $a_{\mathcal{P}}$

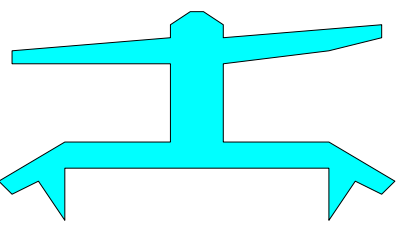

(b) Sample Part

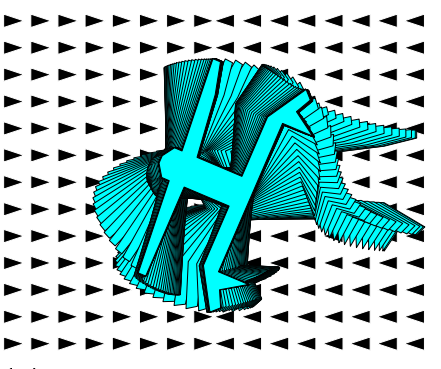

(d) Alignment Task
Figure 8: Determining Rest Configurations

Given a turn function $t_{\mathcal{P}}$ we can define a corresponding alignment function $a_{\mathcal{P}}$ that maps each orientation to a stable orientation. Consider Figure $8 \mathrm{a}$ and c: Stable orientations $\theta_{0}$ act as attractors for all orientations $\theta<\theta_{0}$ for which $t_{\mathcal{P}}(\theta)>0$, and for all $\theta>\theta_{0}$ for which $t_{\mathcal{P}}(\theta)<0$. Thus $a_{\mathcal{P}}$ intersects the diagonal in rest configurations. Metastable orientations act as repellers, corresponding to steps in the graph of $a_{\mathcal{P}}$.

Figure $8 \mathrm{~d}$ traces the part motion in a simulation Note how the part aligns with the node at an angle of $70^{\circ} \approx 1.2$, as predicted by the alignment function $a_{\mathcal{P}}(0) \approx 1.2$ in Figure $8 \mathrm{c}$.

For a more detailed discussion of turn and alignment functions refer to Goldberg [9] and Böhringer et al. [1]. 


\subsection{Extension to Arbitrary Force Fields}

In the previous example the simple form of the force field $f$ yielded simple conditions for rest configurations for part $\mathcal{P}$. If $\mathcal{P}$ is polygonal, there exist explicit formulas for the turn and alignment functions. Different force fields induce different turn and alignment functions. E.g. if $f$ is linear in the distance from the node, force equilibrium is obtained when $\int_{\mathcal{S}} x d A=0$, which implies that the first moment of inertia of the left and right sections of $\mathcal{P}$ have to be equal.

In general vibrations may induce more complex force fields $f$. For each such $f$ the alignment function $a_{\mathcal{P}}$ will be a map from the configuration space to a set of attracting fixed points. The node shapes have to be acquired by the system in a calibration step (e.g. using theoretical methods, or experimentally like Chladni). Once $f$ is known the alignment function $a_{\mathcal{P}}$ can be computed for any given part $\mathcal{P}$. Our current system uses simulation for this task. A more systematic approach using the convolution of the force field $f$ with the contact surface $\mathcal{S}$ is under investigation.

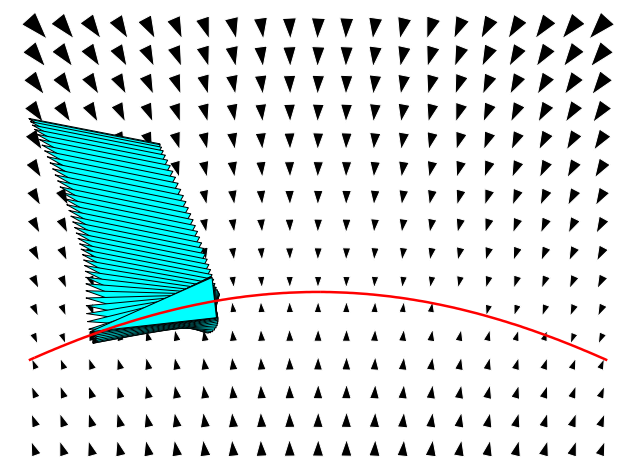

Figure 9: Part Alignment on Vibrating Plate

\subsection{Comparison with Experiments}

Figure 9 shows the simulation of an experiment similar to the one in Figure 3 . Here we model the node as a hyperbolic curve such that the magnitude of $f$ increases proportional with the distance to the node. This is an approximation to the sinusoidal increase in $f$ predicted in the Appendix. Linear approximation is valid because we are primarily concerned with the alignment properties of the part in the vicinity of the node.

The observed rest configurations in experiment and simulation coincide closely. We conclude that to determine the rest configurations we can approximate $f$ with a linear function, which allows the use of analytical methods to determine rest configurations.
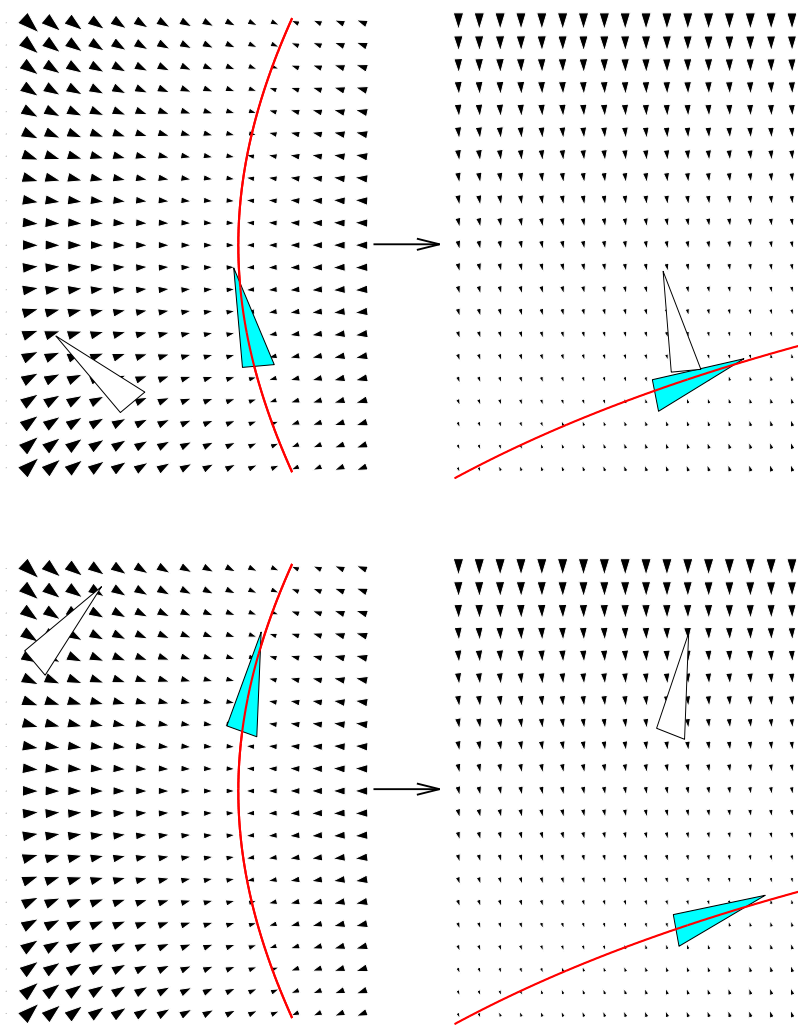

Figure 10: Alignment Plan

\section{Sensorless Part Alignment Plans}

In the previous section we have described how our device aligns parts with vibratory nodes by an openloop manipulation strategy. Our goal is now to bring parts into a specific configuration from where they can be picked up for further processing. In the following we will explain how our device can achieve this task without the use of sensors. Furthermore it is possible to generate and execute these plans automatically by the control software.

As Chladni observed, a wide variety of node shapes can be generated by changing the frequency of the vibrating plate. In addition, clamps can be employed as removable fixtures to generate other types of nodes. A single vibration of the plate reduces the set of possible configurations of a part to a (usually one-dimensional) subset. Subsequent application of different appropriate vibrations can further reduce the size of this subset. Thus we obtain sensorless part alignment plans that are similar to the robot grasp plans developed by Goldberg [9], and the actuator array plans described by Böhringer et al. [1], and Will and Liu [26]. Figure 10 shows such a two-step plan for a triangular part in two different initial configurations. For each 
step, the initial position is drawn in white, and the final position is drawn dark. Note how after two steps the parts reach approximately the same rest configuration.

In his analysis for robot grasp plans, Goldberg [9] shows that any polygonal part can be aligned up to symmetry by such a sensorless alignment plan. Chen and Ierardi [5] showed that the length of the plan is linear in the number of rest configurations of the part.

\section{$7 \quad$ Extensions and Future Work}

Further experiments need to be done to study the effect of surface geometry and friction properties on the final part configuration. The analysis can be further developed to determine the nodes and the vibration mode shape for a given plate setup, and the information used in conjunction with the part geometry and surface property to predict the part behavior. We also propose an extension of the experimental setup by adding software controlled clamps to alter the node shapes in a systematic manner. This will then be combined with automatic calibration of the setup by determining nodes using edge detection, and a planner to automatically generate a plan to get a given part in a desired orientation. Other manipulation tasks like sorting and separating parts of different shapes (as proposed in [26]) are also of great interest. A detailed experimental investigation of the device over a range of parameters and varied operating conditions, combined with development of planning tools is required to make this a viable alternative to existing part feeders.

\section{Appendix}

\section{Particle Bouncing on a Vibrating String}

To understand the effective forces on particles on a vibrating surface, we look at the more tractable case of the planar motion of a particle bouncing on a string in transverse vibrations (Figure 11).

The string vibrates in the first mode, and is not affected by its interaction with the particle. The shape of the string, at time $t$, for a given $x$ location is:

$$
y_{s}=A \sin x \sin 2 \pi \nu t
$$

where $\nu$ is the frequency of oscillation. The position of the particle is given by $\left(x_{p}, y_{p}\right)$.

The interaction between the particle and the string is through a sequence of impacts. We use a model for particle impact with a finite friction coefficient $\mu$, and a coefficient

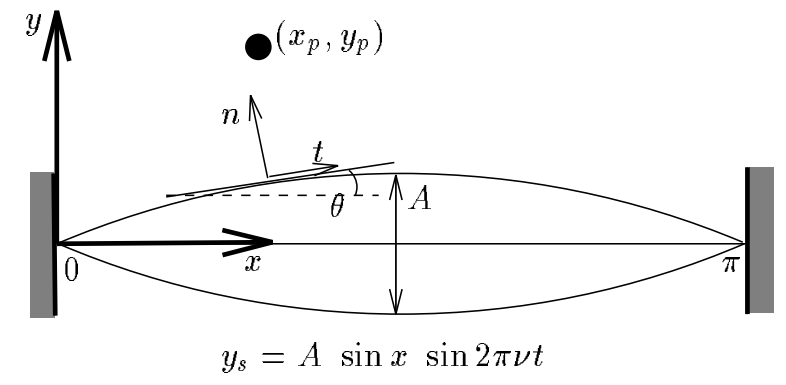

Figure 11: Particle bouncing on a vibrating string.
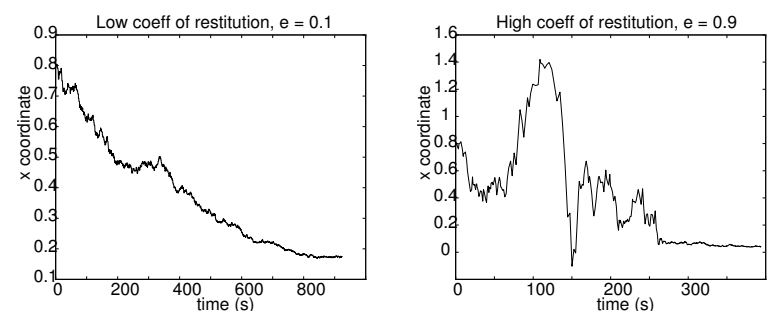

Figure 12: Simulation results showing the position of a particle moving on a vibrating string.

of restitution $e$. $\theta$ is the slope of the string at the point and instant of impact, and is small for small amplitudes of string vibration.

$$
\tan \theta=A \cos x \sin 2 \pi \nu t
$$

The motion of the particle can be simulated as a series of impacts with the string, with the particle in free flight in-between. The change in the momentum of the particle during impact, is calculated using a simple planar impact model. Figure 12 shows the results of a numerical simulation of the model at two different values of $e$.

For a particle starting at rest, at $t=0$, we find that $\dot{y}_{p} \gg \dot{x}_{p}$. Using the assumption that the amplitude of oscillations is small, $\sin \theta \approx \tan \theta, \cos \theta \approx 1$. If $\left(\dot{x}_{p}^{-}, \dot{y}_{p}^{-}\right)$ represent the velocity just before impact, the velocity just after impact $\left(\dot{x}_{p}^{+}, \dot{y}_{p}^{+}\right)$, is:

$$
\begin{aligned}
& \dot{x}_{p}^{+}=e\left(\dot{y}_{p}^{-}-\dot{y}_{s}^{-}\right) \sin \theta+\alpha v_{r e l_{t}}^{-} \\
& \dot{y}_{p}^{+}=\dot{y}_{s}^{-}(1+e)-e \dot{y}_{p}^{-}
\end{aligned}
$$

where $v_{r e l_{t}}^{-}=\left(\dot{y}_{p}^{-}-\dot{y}_{s}^{-}\right) \sin \theta+\dot{x}_{p}^{-}$, is the relative velocity along the tangential direction before impact, and $\alpha \in[0,1]$ is the dissipation factor that depends on $\mu$.

After the impact, $\dot{x}_{p}^{+}$is a sum of the relative tangential velocity before impact, attenuated by friction; and a component from the impulse in the normal direction, which depends on $e$ and the slope of the string at the point of impact. The portion of $x$ impulse added purely due to the effect of the string can be approximated as $-e \dot{y}_{s} \sin \theta$, by setting $\dot{y}_{p}^{-}=0$.

If this component of the impulse were spread uniformly over time, the effective force, $F_{\text {eff }}$, that the particle would experience is:

$$
F_{\text {eff }} \propto-\nu e A^{2} \sin x \cos 2 \pi \nu t \cos x \sin 2 \pi \nu t
$$


We now use the argument that it is more probable for the particle to impact the string at times when the string is above the mean rest position, to show that over a large number of impacts, the time dependent terms in equation (10) average out to a positive quantity. Therefore, the time averaged effective force, $F_{\text {avg }}$, experienced by the particle is:

$$
F_{\text {avg }} \propto-\nu e A^{2} \sin 2 x
$$

This confirms the intuition and the observed behavior that the particle moves faster at higher amplitudes of string oscillation, coefficient of restitution, and oscillation frequency. The sine dependency of the force with $x$ ensures that it points towards the corresponding nodes on either side of the anti-node at $x=\frac{\pi}{2}$.

\section{Acknowledgments}

We thank Subhas Desa, Bruce Donald, and Andy Ruina for useful discussions and valuable comments. Support for the first author is provided in part by the NSF under grants No. IRI-8802390, IRI-9000532, IRI-9201699, and by a Presidential Young Investigator award to Bruce Donald, in part by NSF/ARPA Special Grant for Experimental Research No. IRI-9403903, and in part by the AFOfSR, the Mathematical Sciences Institute, Intel Corporation, and AT\&T Bell laboratories. The second author is supported by a fellowship endowed by the Olin Foundation. The third author is supported by the NSF under Award IRI-9123747 and by National Young Investigator Award IRI-9457523.

\section{References}

[1] K.-F. Böhringer, B. R. Donald, R. Mihailovich, and N. C. MacDonald. Sensorless manipulation using massively parallel microfabricated actuator arrays. In Proc. IEEE Int. Conf. on Robotics and Automation, pages 826-833, San Diego, CA, May 1994.

[2] K.-F. Böhringer, B. R. Donald, R. Mihailovich, and N. C. MacDonald. A theory of manipulation and control for microfabricated actuator arrays. In Proc. IEEE Workshop on Micro Electro Mechanical Systems, pages 102-107, Oiso, Japan, Jan. 1994.

[3] G. Boothroyd, C. Poli, and L. E. Murch. Automatic Assembly. Marcel Dekker, Inc., 1982.

[4] M. E. Caine. The Design of Shape from Motion Constraints. PhD thesis, Massachusetts Institute of Technology, Department of Computer Science, 1993.

[5] Y.-B. Chen and D. Ierardi. The complexity of nonadaptive plans for orienting polygonal parts. Technical Report USC-CS-92-502, University of Southern California, December 1991.

[6] E. F. Chladni. Entdeckungen über die Theorie des Klanges. Weidmanns Erben und Reich, Leipzig, 1787.
[7] M. A. Erdmann and M. T. Mason. An exploration of sensorless manipulation. IEEE Journal of Robotics and Automation, 4(4), Aug. 1988.

[8] G. T. Farnum and B. Davis. Delivering the part. Manufacturing Engineering, March 1986.

[9] K. Y. Goldberg. Orienting polygonal parts without sensing. Algorithmica, 10(2/3/4):201-225, August/September/October 1993.

[10] D. D. Grossman and M. W. Blasgen. Orienting mechanical parts by computer-controlled manipulator. IEEE Trans. on Systems, Man, and Cybernetics, 5, 1975

[11] J. Hartley. Picking parts from a bowlfeeder with image sensing. Sensor Review, 1(1), January 1981.

[12] J. W. Hill. Programmable bowl feeder design based on computer vision. Assembly Automation, 1(1), November 1980

[13] H. Hitakawa. Advanced parts orientation system has wide application. Assembly Automation, 8(3), 1988.

[14] P. Moncevicz, M. Jakiela, and K. Ulrich. Orientation and insertion of randomly presented parts using viratory agitation. In ASME 3rd Conference on Flexible Assembly Systems, September 1991.

[15] J. L. Nevins and D. E. Whitney. Computer-controlled assembly. Scientific American, 1978.

[16] D. Pherson. Programmable feeder for non-rotational parts. In 15th CIRP International Seminar on Manufacturing Systems, 1983.

[17] J. W. S. Rayleigh. The Theory of Sound. Dover, New York, 2nd edition, 1945.

[18] A. H. Redford and E. Lo. Robots in Assembly. Halsted Press, New York, 1986.

[19] F. J. Riley. Assembly Automation, A Management Handbook. Industrial Press, New York, 1983.

[20] B.-Z. Sandler. Robotics: Designing the Mechanisms for Automated Machinery. Prentice Hall, 1991.

[21] B. J. Schroer. Electronic parts presentation using vibratory bowl feeders. Robotics, 3, 1987.

[22] N. C. Singer and W. P. Seering. Utilizing dynamic stability to orient parts. Journal of Applied Mechanics, 54:961-966, Dec. 1987.

[23] T. Suzuki. An approach to a flexible part-feeding system. In 1st International Conference on Assembly Automation, 1980.

[24] S. Timoshenko. Theory of Plates and Shells. McGrawHill, New York, 1940.

[25] S. S. M. Wang and P. Will. Sensors for computer controlled mechanical assembly. Industrial Robot, March 1978 .

[26] P. Will and C. Liu. Parts manipulation on a MEMS intelligent motion surface. Technical Report ISI/RR94-391, USC ISI, Los Angeles, CA, May 1994. 